\title{
An Improved Micromechanical Framework for Saturated Concrete Repaired by the Electrochemical Deposition Method considering the Imperfect Bonding
}

\author{
Qing Chen, ${ }^{1,2}$ Zhengwu Jiang, ${ }^{1}$ Hehua Zhu, ${ }^{3,4}$ J. Woody Ju, ${ }^{5}$ \\ Zhiguo Yan, ${ }^{3,4}$ and Yaqiong Wang ${ }^{2}$ \\ ${ }^{1}$ Key Laboratory of Advanced Civil Engineering Materials, Ministry of Education, Tongji University, 4800 Cao'an Road, \\ Shanghai 201804, China \\ ${ }^{2}$ Shaanxi Provincial Major Laboratory for Highway Bridge and Tunnel, Chang'an University, Xian, Shaanxi 710064, China \\ ${ }^{3}$ State Key Laboratory for Disaster Reduction in Civil Engineering, Tongji University, 1239 Siping Road, Shanghai 200092, China \\ ${ }^{4}$ Key Laboratory of Geotechnical and Underground Engineering, Ministry of Education, Tongji University, 1239 Siping Road, \\ Shanghai 200092, China \\ ${ }^{5}$ Department of Civil and Environmental Engineering, University of California, Los Angeles, CA 90095, USA
}

Correspondence should be addressed to Zhengwu Jiang; jzhw@tongji.edu.cn

Received 12 May 2016; Accepted 21 July 2016

Academic Editor: Song Han

Copyright (C) 2016 Qing Chen et al. This is an open access article distributed under the Creative Commons Attribution License, which permits unrestricted use, distribution, and reproduction in any medium, provided the original work is properly cited.

\begin{abstract}
The interfaces between the deposition products and concrete are not always well bonded when the electrochemical deposition method (EDM) is adopted to repair the deteriorated concrete. To theoretically illustrate the deposition healing process by micromechanics for saturated concrete considering the imperfect interfaces, an improved micromechanical framework with interfacial transition zone (ITZ) is proposed based on our recent studies. In this extension, the imperfect bonding is characterized by the ITZ, whose effects are calculated by modifying the generalized self-consistent model. Meanwhile, new multilevel homogenization schemes are employed to predict the effective properties of repaired concrete considering the ITZ effects. Moreover, modification procedures are presented to reach the properties of repaired concrete with ITZs in the dry state. To demonstrate the feasibility of the proposed micromechanical model, predictions obtained via the proposed micromechanical model are compared with those of the existing models and the experimental data, including results from extreme states during the EDM healing process. Finally, the influences of ITZ and deposition product on the healing effectiveness of EDM are discussed based on the proposed micromechanical model.
\end{abstract}

\section{Introduction}

Over the past 20 years, many meaningful studies have been published on the electrochemical deposition method (EDM), which is a promising repairing approach for concrete in aqueous environment and has been applied to marine structures and other situations in which traditional repairing methods are limited [1-4]. The current literatures mainly focus on the experimental research, including specimen production [5-7], factors influencing the healing effectiveness of EDM [8-10], and assessments of the healing effectiveness of EDM [11-14]. However, there are few theoretical models pertaining to mechanical properties during the EDM healing process. Recently, the authors present micromechanical frameworks to quantitatively describe the mechanical performance of EDM when it is used to heal cracked saturated concrete [1518]. In these micromechanical models, the repaired saturated concrete is represented as three-phase composite composed of the intrinsic concrete, deposition products, and the water phase. During the healing process, the water phase will be replaced by the deposition products. Meanwhile, the effective medium methods, such as the self-consistent approach, the differential scheme, and the Mori-Tanaka method, are 
adopted to estimate the effective properties of concrete during the healing process.

It is noted that the interfaces between the deposition products and the intrinsic concrete are assumed to be well bonded in previous models [15-18]. However, many experimental investigations have shown that the properties of the interfaces between the deposition products and the intrinsic concrete matrix are influenced by many factors, like the solution type and the current density $[8-10,12,13]$, which implies that the perfect bonding assumption is not held reasonable all the time. In this extension, an improved micromechanical model is proposed by considering the imperfect bonding between the deposition product and the intrinsic concrete. The imperfect bonding is characterized by the interfacial transition zone (ITZ), whose effects are calculated by modifying the generalized self-consistent model. Instead of the effective medium method, the noninteraction solution of Ju and Chen [19] is modified to consider the effects of equivalent particle composed of water, deposition product, and ITZ. Meanwhile, new multilevel homogenization schemes considering the ITZ effects are employed to predict the effective properties of repaired concrete. Moreover, modification procedures are presented to reach the properties of repaired concrete with ITZ in the dry state.

Based on our recent works $[15,18]$, this paper presents an improved micromechanical framework to describe the healing process of saturated concrete considering the imperfect bonding between the deposition products and the concrete matrix. An outline of this paper is as follows: Section 2 illustrates the "noninteracting solutions" for the effective properties of two-phase composite. In Section 3, an improved micromechanical model is presented for the healed saturated concrete with imperfect bonding characterized by the ITZ. New multilevel homogenization procedures are proposed to estimate the effective properties of the healed concrete by taking the imperfect bonding into consideration in Section 4. Meanwhile, micromechanical procedures considering the ITZ effects are performed to modify the properties of repaired concrete in the dry state. Numerical examples including experimental validations and comparisons with existing micromechanical models are presented in Section 5, which also discusses the influences of the ITZ and deposition product on the healing effectiveness of EDM based on our proposed micromechanical framework. And some conclusions are reached in Section 6.

\section{The "Noninteracting Solutions" for the Effective Properties of Two-Phase Composite}

2.1. The Effective Properties of the Composite. One goal of continuum micromechanics is to estimate the effective elastic properties of the material defined on the representative volume element (RVE). The RVE is based on a "mesoscopic" length scale which is much larger than the characteristic length scale of particles (inhomogeneities) but smaller than the characteristic length scale of a macroscopic specimen [19]. Taking a two-phase composite as an example, the effective elastic stiffness tensor $\mathbf{C}_{*}$ of the composite is defined through the following:

$$
\overline{\boldsymbol{\sigma}}=\mathrm{C}_{*}: \bar{\varepsilon}
$$

with

$$
\begin{aligned}
\overline{\boldsymbol{\sigma}} & \equiv \frac{1}{V} \int_{V} \boldsymbol{\sigma}(\mathbf{x}) d \mathbf{x}=\frac{1}{V}\left[\int_{V_{m}} \boldsymbol{\sigma}(\mathbf{x}) d \mathbf{x}+\int_{V_{i}} \boldsymbol{\sigma}(\mathbf{x}) d \mathbf{x}\right] \\
\overline{\boldsymbol{\varepsilon}} & \equiv \frac{1}{V} \int_{V} \boldsymbol{\varepsilon}(\mathbf{x}) d \mathbf{x}=\frac{1}{V}\left[\int_{V_{m}} \boldsymbol{\varepsilon}(\mathbf{x}) d \mathbf{x}+\int_{V_{i}} \boldsymbol{\varepsilon}(\mathbf{x}) d \mathbf{x}\right]
\end{aligned}
$$

where $V$ is the volume of an RVE, $V_{m}$ is the volume of the matrix, and $V_{i}$ is the volume of the inhomogeneity. $\overline{\boldsymbol{\varepsilon}}$ and $\overline{\boldsymbol{\sigma}}$ are the volume-average strain and stress of the composite.

\subsection{The "Noninteracting Solutions" for the Effective Properties.} The effective stiffness tensor of two-phase composite containing spherical inhomogeneities can be derived through [19-21]

$$
\mathbf{C}_{*}=\left[\mathbf{C}_{m}+\phi_{i}\left(\mathbf{C}_{i}-\mathbf{C}_{m}\right): \mathbf{B}\right]
$$

where $\mathbf{C}_{*}$ is the effective elastic stiffness tensor of the composite; $\mathbf{C}_{m}$ and $\mathbf{C}_{i}$ are the elastic stiffness tensor of the matrix phase and the inhomogeneity, respectively; $\mathbf{B}$ is the stain concentration tensor; $\phi_{i}$ is the volume fraction of the inhomogeneity. According to our previous work, when the noninteraction solutions are considered, the "strain concentration tensor" can be defined through [21, 22]

$$
\mathbf{B}=[(\mathbf{I}-\mathbf{S H})(\mathbf{A}+\mathbf{S}) \mathbf{A}]^{-1},
$$

with

$$
\begin{aligned}
& \mathbf{H}=\phi_{i}(\mathbf{A}+\mathbf{S})^{-1}, \\
& \mathbf{A} \equiv\left(\mathbf{C}_{i}-\mathbf{C}_{m}\right)^{-1}: \mathbf{C}_{m},
\end{aligned}
$$

where $\mathbf{S}$ is Eshelby's tensor, which depends on the properties of the matrix and the shape of the inclusions; I defines the fourth-order isotropic identity tensor.

\section{Micromechanical Model for Saturated Concrete Repaired Using EDM with the Imperfect Bonding}

3.1. The Micromechanical Approaches to Represent the Imperfect Bonding. There are mainly two analytical models to represent the imperfect bonding between the inclusion phase and matrix phase [23-27]. One is the spring layer model, also known as the interface model, which involves a very thin interfacial zone of unspecified thickness [23-25]. The other is the interphase model, which describes the interfacial zone as a layer between particles and matrix of a specified thickness and of elastic constants different from those of the matrix and the particles $[26,27]$. In this paper, the interphase model is utilized to represent the imperfect bonding between the deposition products and the intrinsic concrete matrix. 


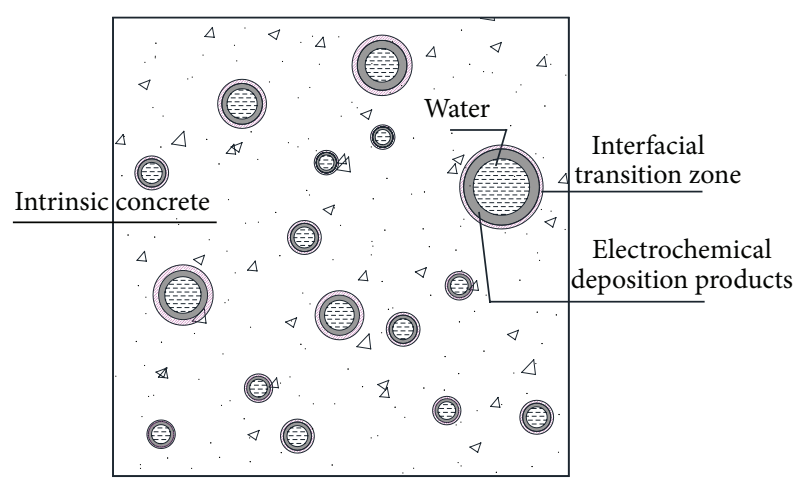

FIGURE 1: Micromechanical model for saturated concrete healed using EDM with interfacial transition zone (ITZ).

3.2. Microstructure of Healed Saturated Concrete with ITZ. The saturated concrete repaired by EDM is described as a multiphase composite composed of pores occupied by water, deposition products, mortar, coarse aggregates, and their interfaces [15-18]. The three traditional solid phases (i.e., mortar, coarse aggregates, and their interfaces) are merged into one matrix phase, namely, intrinsic concrete, in representative volume element [15-18]. Deposition products and water are considered as different inclusion phases in the saturated concrete. Furthermore, in this extension, the interfacial transition zone (ITZ) is considered with the interphase model to characterize the imperfect bonding phenomenon between the deposition product and the intrinsic concrete [8$10,12,13]$.

\subsection{Micromechanical Model for Saturated Concrete Healed} Using EDM with the ITZ. By representing the imperfect bonding with the ITZ, an improved micromechanical model for saturated concrete repaired by EDM can be proposed based on our previous work, which is exhibited in Figure 1. The matrix phase is the intrinsic concrete composed of mortar, coarse aggregates, and their interfaces. The inclusions contain the water, deposition products, and the ITZ. The saturated microcracks and microvoids in the concrete are supposed to be spheres, and the volume of the deposition product is assumed to be proportional to that of each spherical pore [15-18]. Since there is the ITZ in our proposed model, the healing process can be divided into two different stages, including the formation of ITZ and deposition product zone (DPZ). By predicting the effective properties of our model, the concrete's mechanical performance during the healing process is revealed theoretically and quantitatively.

\section{Estimating the Effective Properties of Saturated Concrete Repaired Using EDM with ITZ}

4.1. Multilevel Homogenization Scheme for Estimating Effective Properties. Previously published studies have shown that a homogenization stepping scheme is an effective way to obtain the effective properties of multi-inclusion composites [2841]. The multiphase micromechanical model used in the present study also employs a multilevel homogenization procedure. First, the equivalent inclusion is obtained by the firstlevel homogenization of the two-phase composite composed of water and deposition products, as shown in Figure 2. Second, the equivalent particle is attained by homogenizing the ITZ and the equivalent inclusion, as shown in Figure 3. Third, the effective properties of the equivalent homogeneous composite are calculated by the homogenization of the twophase composite composed of equivalent particles and the intrinsic concrete matrix, as shown in Figure 4.

4.2. The First-Level Homogenization for the Equivalent Inclusion Composed of Water and Deposition Products. The threephase sphere model presented by Christensen and Lo [42], that is, the generalized self-consistent model, is employed to conduct the first-level homogenization by modifying its inner- and outer-layer phases into the water phase and the deposition products, respectively. Let $K_{1}$ and $\mu_{1}\left(K_{2}\right.$ and $\mu_{2}$; $K_{F}$ and $\mu_{F}$ ) denote the bulk modulus and shear modulus of the water (deposition products and equivalent inclusions), respectively. The effective bulk modulus and shear modulus for the equivalent inclusion can be expressed by (7) and (8) as follows:

$$
\begin{aligned}
& K_{F}=K_{2}+\frac{\phi_{F w}\left(K_{1}-K_{2}\right)\left(3 K_{2}+4 \mu_{2}\right)}{3 K_{2}+4 \mu_{2}+3\left(1-\phi_{F w}\right)\left(K_{1}-K_{2}\right)}, \\
& A\left(\frac{\mu_{F}}{\mu_{2}}\right)^{2}+B\left(\frac{\mu_{F}}{\mu_{2}}\right)+C=0
\end{aligned}
$$

with

$$
\phi_{F w}=\frac{V_{\text {wat }}}{V_{\text {wat }}+V_{\text {dep }}},
$$

where $A$ and $B$ are parameters depending on the properties of deposition product and water. See details for our previous work [15]. $\phi_{F w}$ is the volume fraction of the water phase in the two-phase composite composed of the water and the deposition products, $V_{\text {wat }}$ denotes the volume of the water, and $V_{\text {dep }}$ signifies the volume of the deposition products.

4.3. The Second-Level Homogenization for the Equivalent Particle Composed of the ITZ and the Equivalent Inclusion. Let $K_{3}, \mu_{3}, v_{3}$, and $V_{\text {itz }}$ represent the bulk modulus, shear modulus, Poisson's ratio, and volume of the ITZ and $K_{S}, \mu_{S}$ signify the bulk modulus and shear modulus of the equivalent particles made up of the ITZ and the equivalent inclusion. $\phi_{\mathrm{Se}}$ is the volume fraction of the equivalent inclusion in the equivalent particle. $K_{S}$ can be reached with (7) by replacing $K_{1}, K_{2}, \mu_{2}, \phi_{F w}$, and $K_{F}$ with $K_{F}, K_{3}, \mu_{3}, \phi_{\text {Se }}$, and $K_{S}$, respectively. $\mu_{S}$ can be similarly calculated by adopting Christensen and Lo's work [42] with the following expressions:

$$
A^{\prime}\left(\frac{\mu_{S}}{\mu_{3}}\right)^{2}+B^{\prime}\left(\frac{\mu_{S}}{\mu_{3}}\right)+C^{\prime}=0,
$$



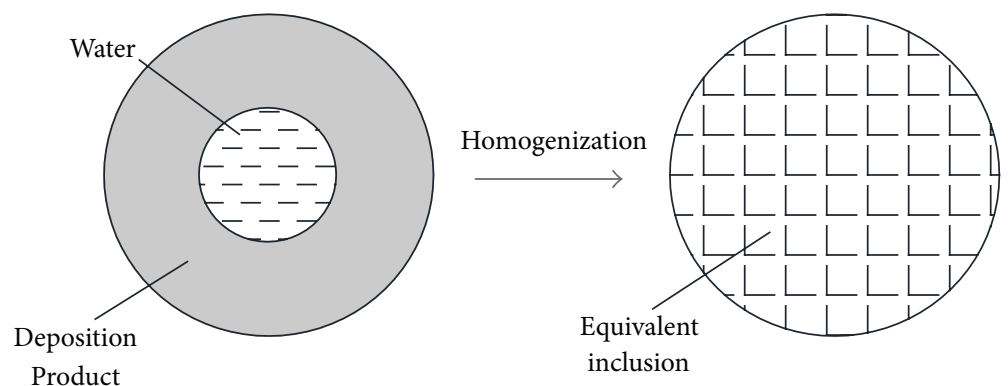

FIGURE 2: The equivalent inclusion obtained by the first-level micromechanical homogenization to the two-phase composite composed of the deposition products and water.

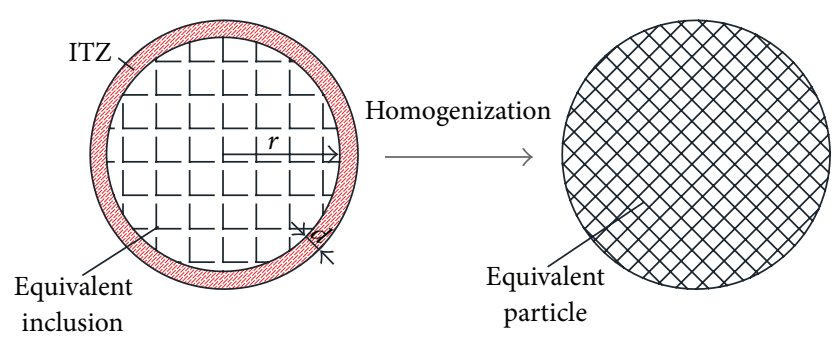

Figure 3: The equivalent particle attained by the second-level micromechanical homogenization to the two-phase composite made up of the ITZ and equivalent inclusion.

where

$$
\begin{aligned}
A^{\prime}= & 8\left[\frac{\mu_{F}}{\mu_{3}}-1\right]\left(4-5 v_{3}\right) \eta_{\alpha} \phi_{\mathrm{Se}}^{10 / 3} \\
& -2\left[63\left(\frac{\mu_{F}}{\mu_{3}}-1\right) \eta_{\beta}+2 \eta_{\alpha} \eta_{\gamma}\right] \phi_{\mathrm{Se}}^{7 / 3} \\
& +252\left[\frac{\mu_{F}}{\mu_{3}}-1\right] \eta_{\beta} \phi_{\mathrm{Se}}^{5 / 3} \\
& -50\left[\frac{\mu_{F}}{\mu_{3}}-1\right]\left(7-12 v_{3}+8 v_{3}^{2}\right) \eta_{\beta} \phi_{\mathrm{Se}} \\
& +4\left(7-10 v_{3}\right) \eta_{\beta} \eta_{\gamma}, \\
B^{\prime}= & -4\left[\frac{\mu_{F}}{\mu_{3}}-1\right]\left(1-5 v_{3}\right) \eta_{\alpha} \phi_{\mathrm{Se}}^{10 / 3} \\
& +4\left[63\left(\frac{\mu_{F}}{\mu_{3}}-1\right) \eta_{\beta}+2 \eta_{\alpha} \eta_{\gamma}\right] \phi_{\mathrm{Se}}^{7 / 3} \\
& -504\left[\frac{\mu_{F}}{\mu_{3}}-1\right] \eta_{\beta} \phi_{\mathrm{Se}}^{5 / 3} \\
& +150\left[\frac{\mu_{F}}{\mu_{3}}-1\right]\left(3-v_{3}\right) v_{3} \eta_{\beta} \phi_{\mathrm{Se}} \\
& 3\left(15 v_{3}-7\right) \eta_{\beta} \eta_{\gamma},
\end{aligned}
$$

$$
\begin{aligned}
C^{\prime}= & 4\left[\frac{\mu_{F}}{\mu_{3}}-1\right]\left(5 v_{3}-7\right) \eta_{\alpha} \phi_{\mathrm{Se}}^{10 / 3} \\
& -2\left[63\left(\frac{\mu_{F}}{\mu_{3}}-1\right) \eta_{\beta}+2 \eta_{\alpha} \eta_{\gamma}\right] \phi_{\mathrm{Se}}^{7 / 3} \\
& +252\left[\frac{\mu_{F}}{\mu_{3}}-1\right] \eta_{\beta} \phi_{\mathrm{Se}}^{5 / 3} \\
& +25\left[\frac{\mu_{F}}{\mu_{3}}-1\right]\left(v_{3}^{2}-7\right) \eta_{\beta} \phi_{\mathrm{Se}} \\
& -3\left(7+5 v_{3}\right) \eta_{\beta} \eta_{\gamma},
\end{aligned}
$$

with

$$
\begin{aligned}
\eta_{\alpha}= & {\left[\frac{\mu_{F}}{\mu_{3}}-1\right]\left(49-50 v_{F} v_{3}\right)+35\left(\frac{\mu_{F}}{\mu_{3}}\right)\left(v_{F}-2 v_{3}\right) } \\
& +35\left(2 v_{F}-v_{3}\right) \\
\eta_{\beta}= & 5 v_{F}\left[\frac{\mu_{F}}{\mu_{3}}-8\right]+7\left[\frac{\mu_{F}}{\mu_{3}}+4\right], \\
\eta_{\gamma}= & \frac{\mu_{F}}{\mu_{3}}\left[8-10 v_{3}\right]+\left(7-5 v_{3}\right), \\
\phi_{\mathrm{Se}}= & \frac{V_{\text {wat }}+V_{\mathrm{dep}}}{V_{\mathrm{itz}}+V_{\mathrm{dep}}+V_{\mathrm{wat}}}
\end{aligned}
$$

where $V_{\text {itz }}$ denotes the volume of ITZ.

4.4. The Third-Level Homogenization for the Equivalent Homogenous Composite Composed of the Equivalent Particle and Intrinsic Concrete. The noninteraction solutions of [19] are adopted to get the effective properties of the repaired concrete. Let $\mathbf{C}_{S}, \mathbf{C}_{4}$, and $\mathbf{C}_{T}$ represent the stiffness tensor of the equivalent particle, the intrinsic concrete, and the equivalent composite of the saturated concrete repaired by EDM with ITZ. For the isotropic matrix and spherical equivalent 


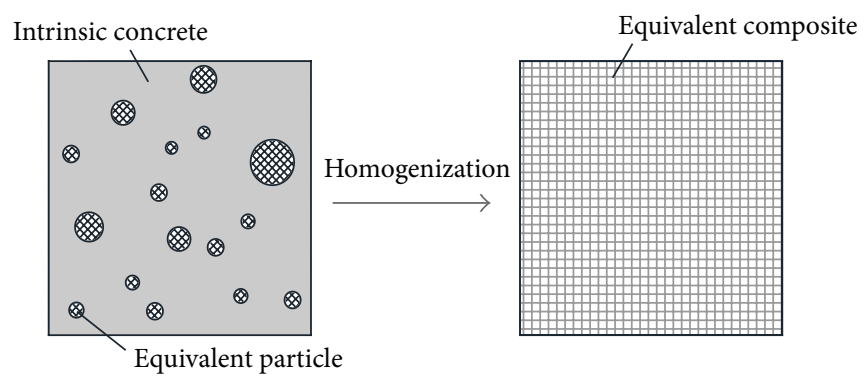

FIgURE 4: The equivalent composite of repaired concrete reached by the third-level micromechanical homogenization to the two-phase composite consisting of the intrinsic concrete and equivalent particle.

inclusions, the tensorial components of $\mathbf{I}, \mathbf{S}, \mathbf{C}_{S}, \mathbf{C}_{4}$, and $\mathbf{C}_{T}$ are as follows:

$$
\begin{aligned}
I_{i j k l}= & \frac{1}{3} \delta_{i j} \delta_{k l}+\frac{1}{2}\left(\delta_{i k} \delta_{j l}+\delta_{i l} \delta_{j k}-\frac{2}{3} \delta_{i j} \delta_{k l}\right), \\
S_{i j k l}= & \frac{K_{4}}{3 K_{4}+4 G_{4}} \delta_{i j} \delta_{k l} \\
& +\frac{3\left(K_{4}+2 G_{4}\right)}{5\left(3 K_{4}+4 G_{4}\right)}\left(\delta_{i k} \delta_{j l}+\delta_{i l} \delta_{j k}-\frac{2}{3} \delta_{i j} \delta_{k l}\right), \\
C_{S i j k l}= & K_{S} \delta_{i j} \delta_{k l}+\mu_{S}\left(\delta_{i k} \delta_{j l}+\delta_{i l} \delta_{j k}-\frac{2}{3} \delta_{i j} \delta_{k l}\right), \\
C_{4 i j k l}= & K_{4} \delta_{i j} \delta_{k l}+\mu_{4}\left(\delta_{i k} \delta_{j l}+\delta_{i l} \delta_{j k}-\frac{2}{3} \delta_{i j} \delta_{k l}\right), \\
C_{T i j k l}= & K_{T} \delta_{i j} \delta_{k l}+\mu_{T}\left(\delta_{i k} \delta_{j l}+\delta_{i l} \delta_{j k}-\frac{2}{3} \delta_{i j} \delta_{k l}\right),
\end{aligned}
$$

where $\delta_{i j}$ is the Kronecker delta. $K_{4}, \mu_{4}$ are, respectively, the bulk modulus and shear modulus of the intrinsic concrete, and $K_{T}, \mu_{T}$ are those of the equivalent composite of the saturated concrete repaired by EDM with ITZ, correspondingly. By replacing the matrix phase and inhomogeneities (inclusion phase) with the intrinsic concrete and the equivalent particle, the Ju and Chen model is modified to obtain the effective properties of the saturated concrete repaired by EDM with ITZ. By substituting (13) into (1)-(5), the effective bulk modulus and shear modulus of the saturated concrete repaired by EDM with ITZ are attained by the following equations after some derivations:

$$
\begin{aligned}
K_{T} & =K_{4}\{1 \\
& \left.+\frac{3\left(1-v_{4}\right)\left(K_{S}-K_{4}\right) \phi_{T p}}{3\left(1-v_{4}\right) K_{4}+\left(1-\phi_{T p}\right)\left(1+v_{4}\right)\left(K_{S}-K_{4}\right)}\right\}, \\
\mu_{T} & =\mu_{4}\{1 \\
& \left.+\frac{15\left(1-v_{4}\right)\left(\mu_{S}-\mu_{4}\right) \phi_{T p}}{15\left(1-v_{4}\right) \mu_{4}+\left(1-\phi_{T p}\right)\left(8-10 v_{4}\right)\left(\mu_{S}-\mu_{4}\right)}\right\},
\end{aligned}
$$

with

$$
\phi_{T p}=\frac{V_{\mathrm{wat}}+V_{\mathrm{dep}}+V_{\mathrm{itz}}}{V_{\mathrm{wat}}+V_{\mathrm{dep}}+V_{\mathrm{itz}}+V_{\mathrm{mat}}}=\frac{V_{\mathrm{wat}}+V_{\mathrm{dep}}+V_{\mathrm{itz}}}{V_{\mathrm{tot}}},
$$

where $V_{\text {mat }}$ and $V_{\text {tot }}$ mean the volume of the intrinsic concrete matrix and the total volume of the composite. With regard to the effect of water viscosity in pores of the saturated concrete [43-46], $\mu_{T}$ should multiply the modification function as below [43]:

$$
F=1+f_{1} \phi_{T p}^{2}+f_{2} \phi_{T p}
$$

where $f_{1}$ and $f_{2}$ are parameters investigated by the experiment [43].

Furthermore, the Young modulus of unsaturated concrete can be obtained based on the theorem of elastic mechanics, provided that the bulk modulus and shear modulus are known. Hence,

$$
E_{T}=\frac{9 K_{T} \mu_{T}}{3 K_{T}+\mu_{T}}
$$

where $E_{T}$ is the Young modulus of the equivalent homogenous composite (i.e., the healed saturated concrete).

4.5. Modifications to Estimations of Effective Properties with ITZ in Dry Conditions. Modifications should be made to our proposed framework when it is applied to estimate the properties of the saturated concrete repaired using EDM in the dry state [15].

Firstly, the properties pertaining to water should be replaced by those pertaining to air, and the water effect should be ignored. Secondly, since the pores should not be assumed to be spherical in the dry state, the similar modification coefficients, $\chi_{K}, \chi_{\mu}$, and $\chi_{E}$, are introduced to reflect the influence of the crack (pore) shape in the following:

$$
\begin{gathered}
\chi_{K}=\frac{K_{\alpha}^{*}}{K_{\alpha=1}^{*}} \\
\chi_{\mu}=\frac{\mu_{\alpha}^{*}}{\mu_{\alpha=1}^{*}}
\end{gathered}
$$




$$
\begin{aligned}
\chi_{E} & =\frac{E_{\alpha}^{*}}{E_{\alpha=1}^{*}} \\
\alpha & =\frac{1}{N} \sum_{i=1}^{N} \frac{a_{i}}{b_{i}},
\end{aligned}
$$

where $K_{\alpha=1}^{*}, \mu_{\alpha=1}^{*}$, and $E_{\alpha=1}^{*}$ are the predicted effective bulk modulus, effective shear modulus, and Young's modulus, respectively, when the crack (pore) shape is spherical ( $\alpha=$ 1). Moreover, $K_{\alpha}^{*}, \mu_{\alpha}^{*}$, and $E_{\alpha}^{*}$ are the predicted effective bulk modulus, effective shear modulus, and Young's modulus, respectively, when the pore shape is not spherical $(\alpha<1)$; $\alpha$ is the equivalent aspect ratio of the pores; $a_{i}$ and $b_{i}$ are the lengths of the pores' minor and major axes, respectively; and $N$ is the number of different pores in the concrete.

The results in [47] are modified to reach the above predicted effective properties. See details for [15]. However, due to the imperfect bonding effect, $K_{2}$ and $\mu_{2}$ in (37)-(43) of [15] should be replaced by $K_{\text {ave }}$ and $\mu_{\text {ave }}$, which are obtained by the following expressions:

$$
\begin{aligned}
& K_{\mathrm{ave}}=0.5\left(\phi_{\mathrm{Md}} K_{2}+\phi_{\mathrm{Ml}} K_{3}+\left(1-\phi_{\mathrm{Md}}-\phi_{\mathrm{Ml}}\right) K_{4}\right) \\
&+ 0.5\left(\frac{1}{\left(\phi_{\mathrm{Md}} / K_{2}+\phi_{\mathrm{Ml}} / K_{3}+\left(1-\phi_{\mathrm{Md}}-\phi_{\mathrm{Ml}}\right) / K_{4}\right)}\right), \\
& \mu_{\mathrm{ave}}= 0.5\left(\phi_{\mathrm{Md}} \mu_{2}+\phi_{\mathrm{Ml}} \mu_{3}+\left(1-\phi_{\mathrm{Md}}-\phi_{\mathrm{Ml}}\right) \mu_{4}\right) \\
&+ 0.5\left(\frac{1}{\left(\phi_{\mathrm{Md}} / \mu_{2}+\phi_{\mathrm{Ml}} / \mu_{3}+\left(1-\phi_{\mathrm{Md}}-\phi_{\mathrm{Ml}}\right) / \mu_{4}\right)}\right), \\
& \phi_{\mathrm{Md}}=\frac{V_{\mathrm{dep}}}{V_{\mathrm{dep}}+V_{\mathrm{itz}}+V_{\mathrm{mat}}}, \\
& \phi_{\mathrm{Ml}}=\frac{V_{\mathrm{itz}}}{V_{\mathrm{dep}}+V_{\mathrm{itz}}+V_{\mathrm{mat}}} .
\end{aligned}
$$

Furthermore, if ultrasound waves are employed to test the effective dynamic properties under dry conditions, the static properties should be modified to dynamic properties according to the relationship between them $[15,48]$.

\section{Verification and Discussion}

5.1. Comparison with the Existing Model and EDM Experiments under Dry Conditions. The predictions in this study are compared with the experimental data and the estimations of the existing models to verify the capacity of the proposed micromechanical framework for saturated concrete repaired by the EDM considering the imperfect bonding.

Firstly, the existing models [15] are utilized to verify our proposed micromechanical framework. The properties of intrinsic concrete and deposition products are from [15]. Four different types of ITZ properties are utilized as examples to perform the simulations, which are listed in Table 1 . The ratio between the volume of ITZ and equivalent inclusion is 0.1 . It is noted that the specific values for the thicknesses and properties of the ITZs are not our central focus here. In this
TABLE 1: Properties of four types of ITZs between the deposition products inclusion and the concrete.

\begin{tabular}{lcc}
\hline & Bulk modulus $(\mathrm{GPa})$ & Shear modulus $(\mathrm{GPa})$ \\
\hline Type 1 & 18.61 & 12.3 \\
Type 2 & $18.61 * 0.8$ & $12.3 * 0.8$ \\
Type 3 & $18.61 * 0.4$ & $12.3 * 0.4$ \\
Type 4 & $18.61 * 0.2$ & $12.3 * 0.2$ \\
\hline
\end{tabular}

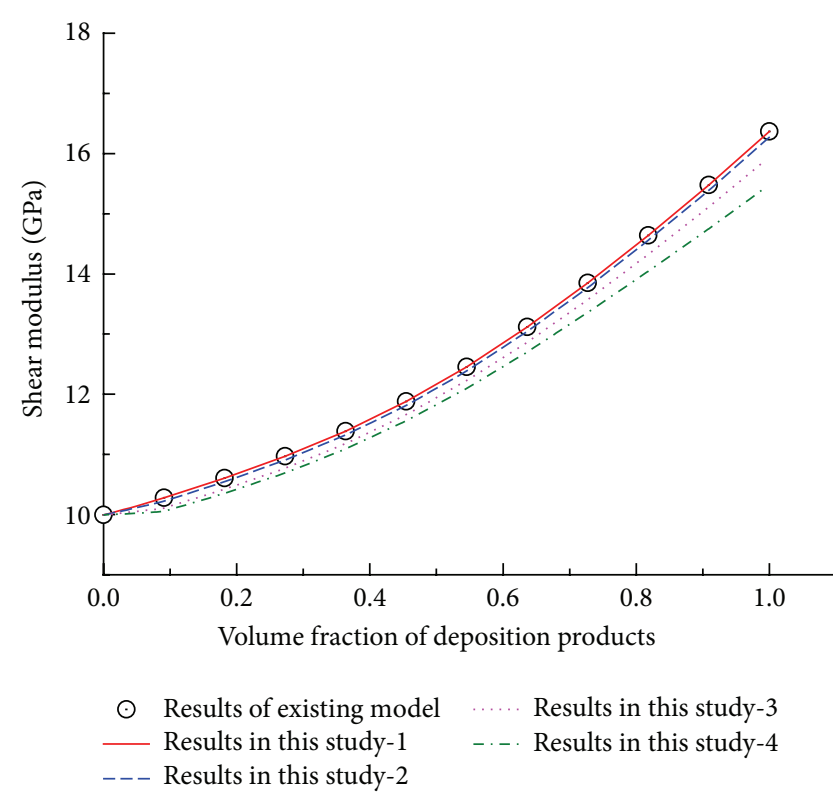

Figure 5: Comparisons of shear modulus between our predictions and those of existing model [15] during healing process, with 1, 2, 3, and 4 representing results obtained with the first, second, third, and fourth types of ITZ.

paper, we instead focus on the quantitative influence of the specified ITZs on the healing effects.

Figure 5 presents the comparisons of mechanical properties between our predictions and those of Zhu et al. [15] during healing process. From Figure 5, it can be observed that our predictions for shear modulus of repaired concrete are the same as those of Zhu et al. [15], when the properties of ITZ are equal to those of the deposition products, which implies that our proposed micromechanical model is capable of describing the healing process of saturated concrete with perfect interface. With the decrease of the IZT properties, the healed specimen demonstrates lower properties. Meanwhile, the results of the two different micromechanical models show that the values of effective shear modulus gradually increase during the healing process due to the accumulation of deposition products. As to the effective Young modulus, similar conclusions can be reached, which are exhibited by Figure 6.

Secondly, the dynamic Young moduli of the specimen before and after healing in Chen's experiment [14] are adopted to validate the proposed micromechanical model with ITZ in the dry state. The average initial porosity of the specimen is 0.299 . The average pulse-velocity of its intrinsic concrete is 


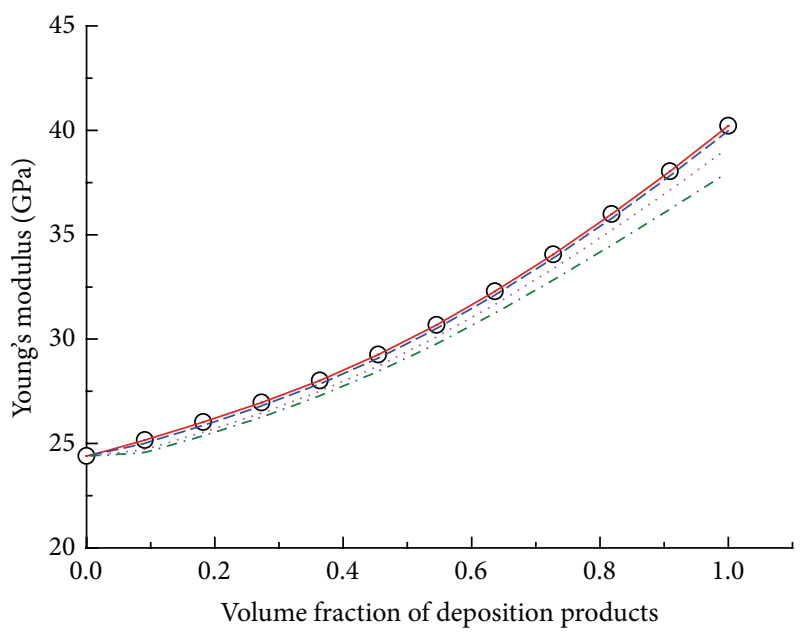

$\begin{aligned} \odot & \begin{array}{l}\text { Results of existing model } \\ -\end{array} \text { Results in this study-1 } \\ - & \ldots . \cdot \text { Results in this study-3 } \\ -- & \text { Results in this study-2 }\end{aligned}$

Figure 6: Comparisons of Young's modulus between our predictions and those of existing model [15] during healing process, with 1 , 2,3 , and 4 representing results obtained with the first, second, third, and fourth types of ITZ.

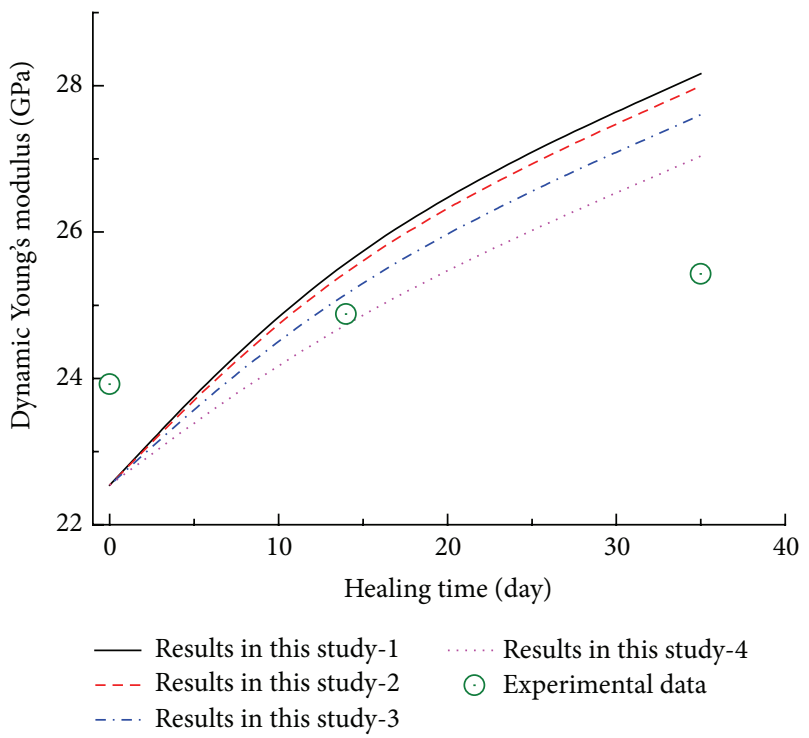

Figure 7: Comparison between the results obtained with the proposed model and those obtained experimentally [14] for the dynamic Young modulus in the dry state, with $1,2,3$, and 4 representing results obtained with the first, second, third, and fourth types of ITZ.

$5134.5 \mathrm{~m} / \mathrm{s}$. The density is $2537.9 \mathrm{~kg} / \mathrm{m}^{3}$ and Poisson's ratio is 0.229. As exhibited in Figure 7, the predictions considering the ITZ effects (i.e., results obtained with type 2, 3, and 4 ITZ properties) correspond better with the experimental data than those without taking ITZ effects into consideration (i.e., results reached with type 1 ITZ properties).

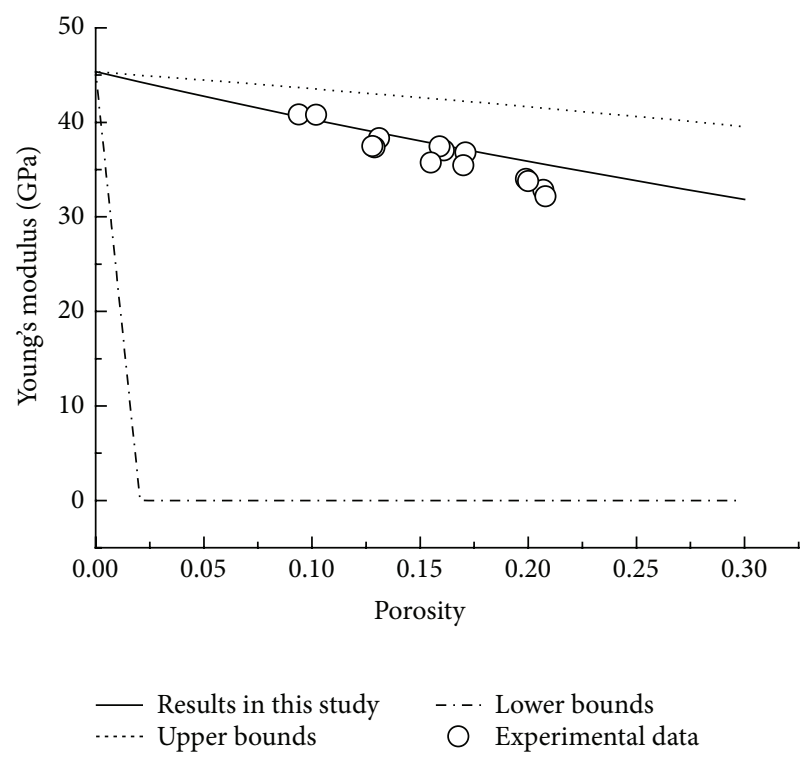

FIGURE 8: The comparison between the results obtained with the different micromechanical models and those obtained experimentally [48] for the static Young modulus of saturated concrete.

Thirdly, if there is absolutely no healing process in the concrete, the proposed model can predict the properties of the saturated concrete. Figure 8 exhibits the comparisons among our results, those obtained by the upper bounds, lower bounds, and the experimental data of Yaman et al. [48]. The comparisons show that the predictions herein agree well with the experimental data. In addition, if the saturated concrete has been completely healed by the EDM, there is no water effect and the healed concrete is effectively a two-phase composite with isotropic spherical inclusion phase with imperfect interfacial bonds. Furthermore, if the properties of ITZ are the same as those of inclusions, which implies that there are perfect interfaces between the inclusions and matrix, the proposed model can predict the two-phase composite with isotropic spherical inclusion phase. The work done by Cohen and Ishai [49] is employed to verify our proposed model at this state. Figures 9-10 present the comparisons among our results, the upper bounds, lower bounds, and the experimental data of Cohen and Ishai [49]. From Figures 9 and 10, it can be found that our predictions correspond with the experimental data well.

5.2. Influences of ITZ Thickness and Electrochemical Deposition Product on the Healing Effectiveness. The ITZ has a significant impact on the effective properties of the composite [23-27]. The volume fraction of ITZ in the equivalent particle can be represented as $\phi_{\mathrm{ITZ}}=1-(r /(r+d))^{3}$ from Figure 3, where $d$ is the ITZ thickness. Since we focus on the quantitative influence of ITZ on the healing effectiveness of EDM, the exact value for $d$ or $\phi_{\text {ITZ }}$ is not the interest of this study. To investigate these effects quantitatively, three different values of $\phi_{\text {ITZ }}$, that is, $0.01,0.1$, and 0.2 , are employed as examples. Figures 11 and 12 present the variations in the shear modulus and Young's modulus of the equivalent composite 


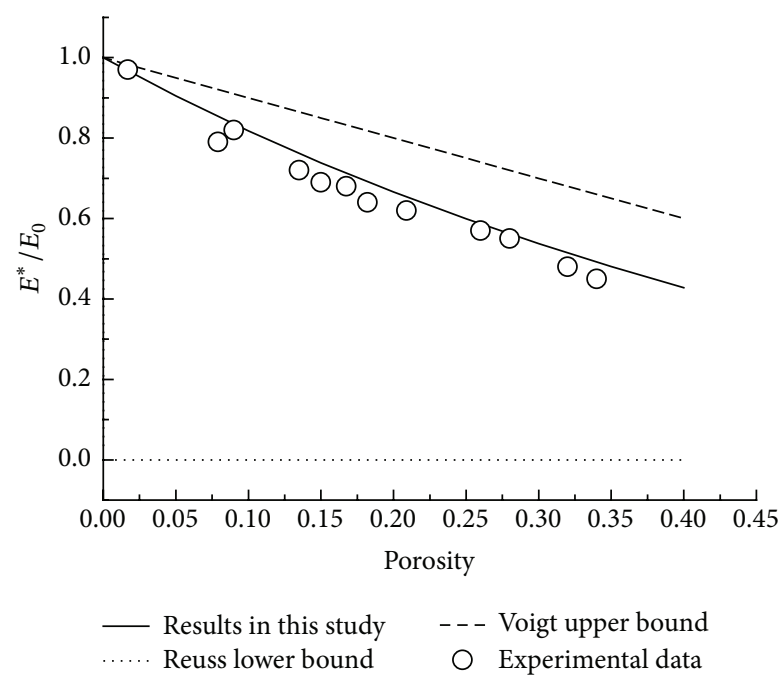

FIGURE 9: The comparison among the results obtained with the proposed micromechanical model, those obtained experimentally [49], those estimated by the Reuss lower bound, and the Voigt upper bound for the effective Young modulus of the porous matrix.

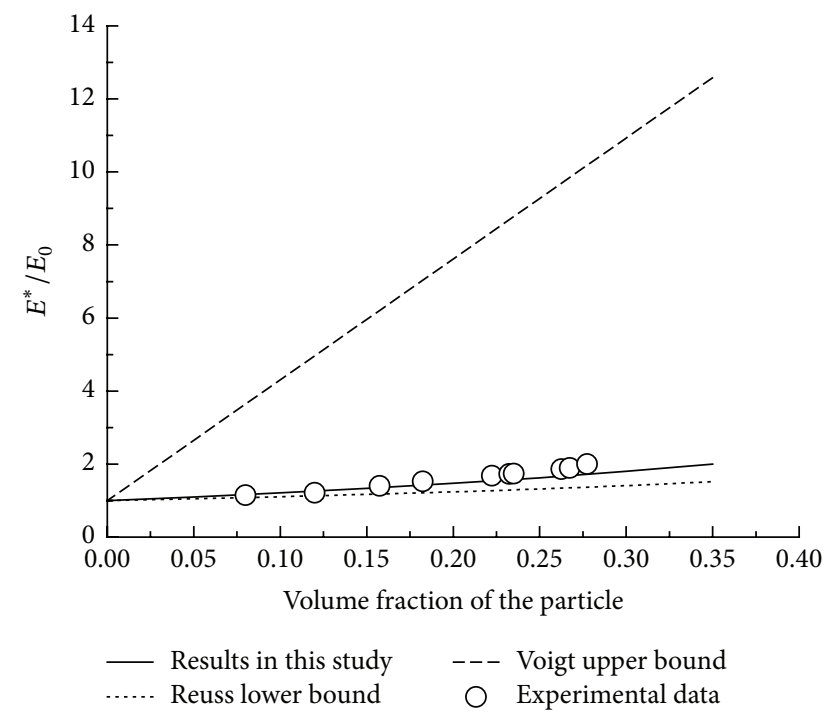

FIGURE 10: The comparison among the results obtained with the proposed micromechanical model, those obtained experimentally [49], those estimated by the Reuss lower bound, and the Voigt upper bound for the effective Young modulus of the nonporous composite.

of the healed saturated concrete with imperfect bonding. The properties of the equivalent composite gradually increase during the healing process. Furthermore, with the increase of the ITZ thickness, the equivalent composite demonstrates lower effective properties.

In addition, three types of deposition products from [15] are utilized as examples to illustrate their influence on the healing effectiveness. In these simulations, $\phi_{\text {ITZ }}=0.1$. From Figure 13, it can be observed that the effective Young modulus increases during the healing process. Before the point of $\phi_{\text {ITZ }}=0.1$, the improvement of Young's modulus is less than

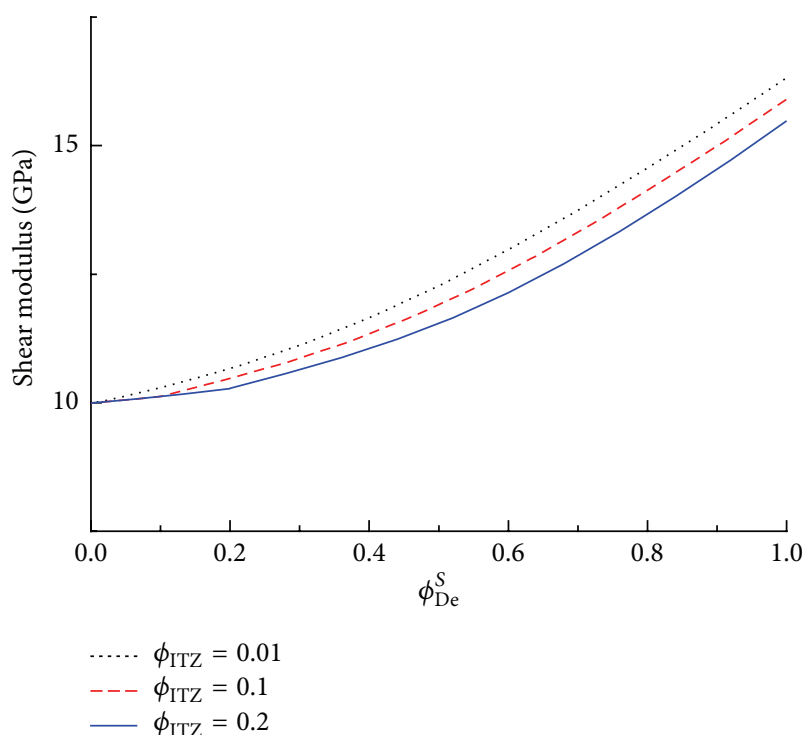

FIGURE 11: Influence of the ITZ on the shear modulus of repaired concrete during the healing process, where $\phi_{\text {ITZ }}$ is the volume fraction of ITZ in the equivalent particle; $\phi_{\mathrm{De}}^{S}$ is the volume fraction of the deposition products in the equivalent particle.

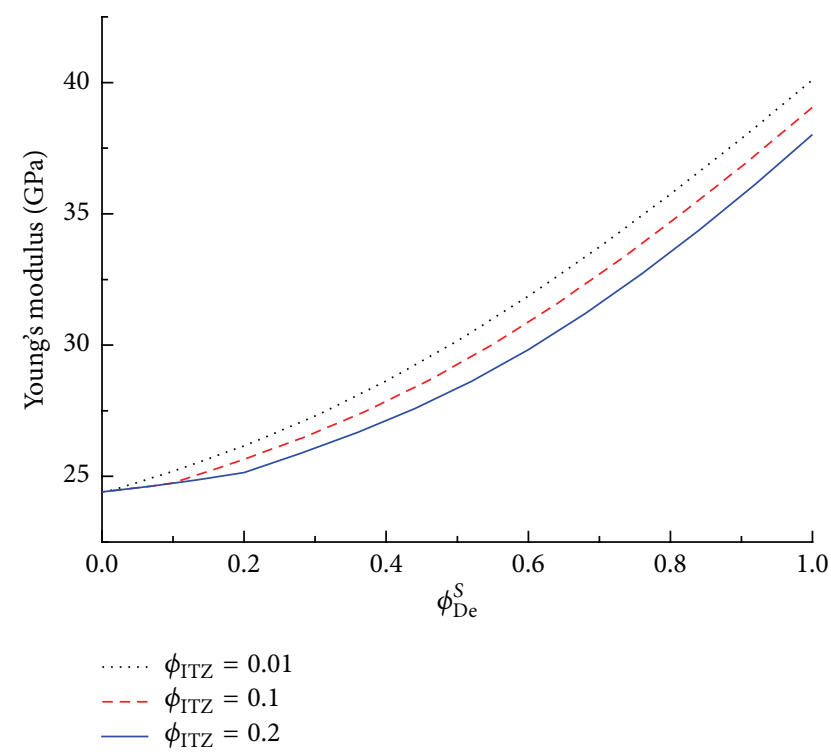

FIGURE 12: Influence of the ITZ on the Young modulus of repaired concrete during the healing process, where $\phi_{\text {ITZ }}$ is the volume fraction of ITZ in the equivalent particle; $\phi_{\mathrm{De}}^{S}$ is the volume fraction of the deposition products in the equivalent particle.

those in the period after that point, because the properties of ITZ are smaller than those of DPZ. When the properties of the deposition products increase, the Young modulus of healed concrete becomes greater. As to the effective shear modulus, similar conclusions can be reached as Figure 14 shows. 


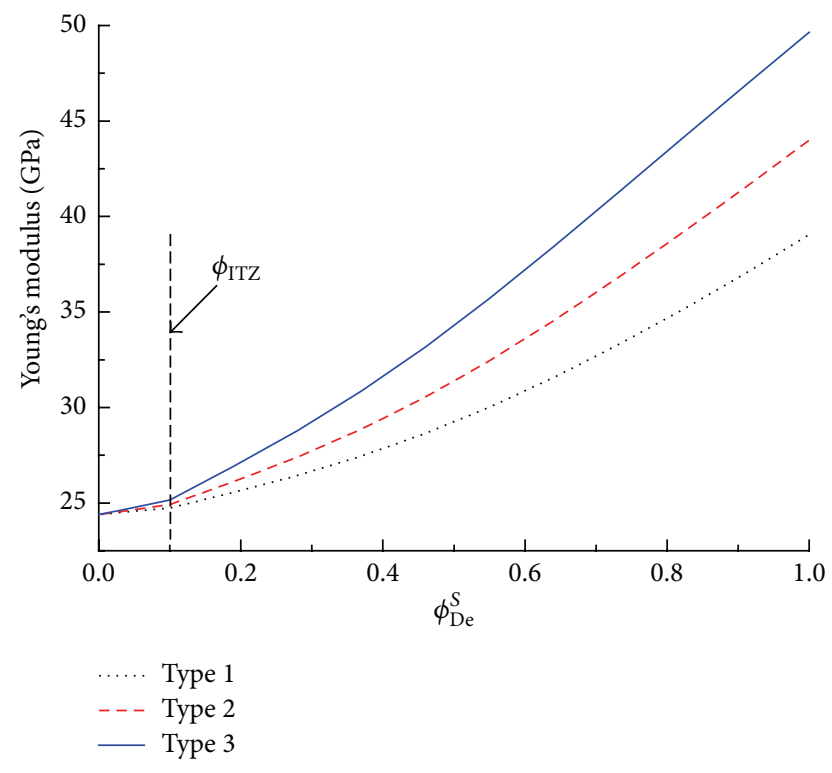

FIGURE 13: Influence of the properties of the deposition products on the Young modulus of repaired concrete during the healing process, where $\phi_{\text {ITZ }}$ is the volume fraction of ITZ in the equivalent particle; $\phi_{\mathrm{De}}^{S}$ is the volume fraction of the deposition products in the equivalent particle.

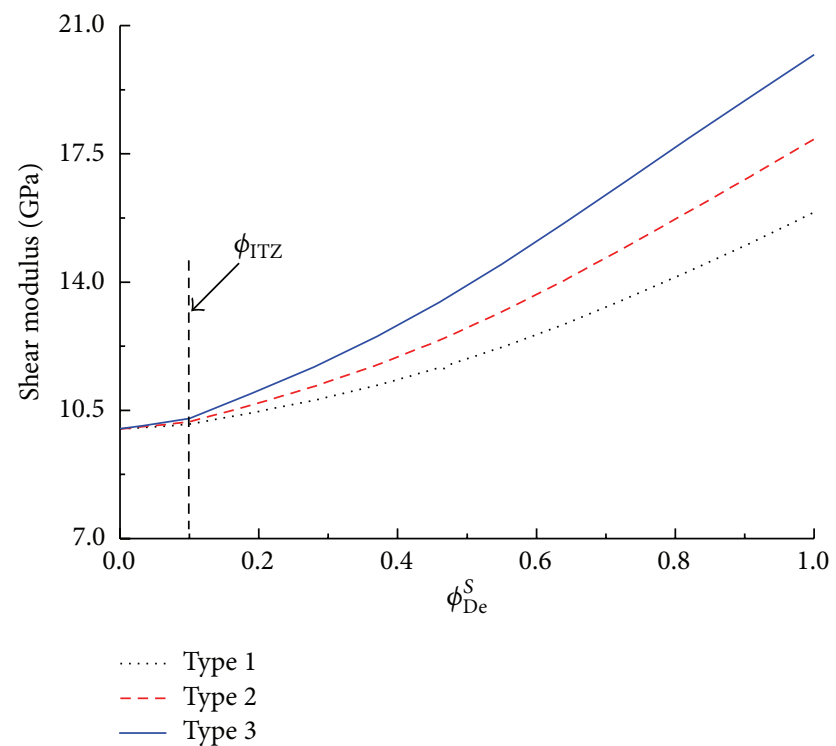

Figure 14: Influence of the properties of the deposition products on the shear modulus of repaired concrete during the healing process, where $\phi_{\text {ITZ }}$ is the volume fraction of ITZ in the equivalent particle; $\phi_{\mathrm{De}}^{S}$ is the volume fraction of the deposition products in the equivalent particle.

\section{Conclusions}

The bonds between the deposition products and concrete are not always perfect when the electrochemical deposition method (EDM) is applied to repair the deteriorated concrete. As an extension of our recent studies, this paper proposes an improved micromechanical model with ITZ to theoretically illustrate the deposition healing process by micromechanics and quantitatively describe the effective properties of saturated concrete repaired by EDM considering the imperfect bonding. To quantitatively consider the ITZ effects, a new multilevel homogenization scheme is proposed by incorporating the generalized self-consistent method and the noninteracting solutions of Ju and Chen's model together. Modification procedures are presented to obtain the properties of repaired concrete with ITZs in the dry state. Moreover, our predicted results are compared to available experimental data and the predictions of existing micromechanical models. The influences of the ITZ and deposition products on the healing effectiveness are discussed based on the proposed micromechanical model. From this study, the following main conclusions can be drawn:

(1) The proposed micromechanical model with ITZ is both feasible and capable of describing the mechanical performance of saturated concrete during the EDM healing process with imperfect bonding.

(2) For the special cases, the proposed model can predict the properties of saturated concrete repaired by EDM with perfect bonding, the properties of saturated concrete, and particle reinforced composite.

(3) Based on our proposed model, the ITZ and deposition products play important roles in the healing effectiveness of EDM.

\section{Competing Interests}

The authors declare that there are no competing interests regarding the publication of this paper.

\section{Acknowledgments}

This work is supported by the National Key Basic Research and Development Program (973 Program, no. 2011CB013800) and National Natural Science Foundation of China (51508404, 51478348, 51278360, and 51308407). This work is also supported by Key Laboratory of Advanced Civil Engineering Materials (Tongji University), Program of Shanghai Science and Technology Commission (15DZ1205003), the 1000 Talents Plan Short-Term Program by the Organization Department of the Central Committee of the CPC, Research Program of State Key Laboratory for Disaster Reduction in Civil Engineering, the Scientific Platform Open Funds of Fundamental Research Plan for the Central Universities, Chang'an University (310821151113), and Natural Science Foundation of Shandong Province (ZR2013EEL019).

\section{References}

[1] M. Yokoda and T. Fukute, "Rehabilitation and protection of marine concrete structure using electrodeposition method," in Proceedings of the International RILEM/CSIRO/ACRA Conference on Rehabilitation of Concrete Structures (RILEM '92), pp. 213-222, Melbourne, Australia, 1992. 
[2] H. Sasaki and M. Yokoda, "Repair method of marine reinforced concrete by electro deposition technique," in Proceedings of the Annual Conference of Japanese Concrete Institute, pp. 849-854, 1992.

[3] Z. W. Jiang, W. T. Li, and Z. C. Yuan, "Influence of mineral additives and environmental conditions on the self-healing capabilities of cementitious materials," Cement and Concrete Composites, vol. 57, pp. 116-127, 2015.

[4] W. T. Li, Z. W. Jiang, Z. H. Yang, N. Zhao, and W. Z. Yuan, "Self-healing efficiency of cementitious materials containing microcapsules filled with healing adhesive: mechanical restoration and healing process monitored by water absorption," PLoS ONE, vol. 8, no. 11, Article ID e81616, 2013.

[5] J. S. Ryou, "New waterproofing technique for leaking concrete," Journal of Materials Science Letters, vol. 22, no. 14, pp. 1023-1025, 2003.

[6] J. S. Ryou and N. Otsuki, "Experimental study on repair of concrete structural members by electrochemical method," Scripta Materialia, vol. 52, no. 11, pp. 1123-1127, 2005.

[7] J.-J. Chang, W. Yeih, H.-M. Hsu, and N.-M. Huang, "Performance evaluation of using electrochemical deposition as a repair method for reinforced concrete beams," Structrual Longevity, vol. 1, no. 2, pp. 75-93, 2009.

[8] N. Otsuki and J.-S. Ryu, "Use of electrodeposition for repair of concrete with shrinkage cracks," Journal of Materials in Civil Engineering, vol. 13, no. 2, pp. 136-142, 2001.

[9] J. S. Ryu, "Influence of crack width, cover depth, water-cement ratio and temperature on the formation of electrodeposits on the concrete surface," Magazine of Concrete Research, vol. 55, no. 1, pp. 35-40, 2003.

[10] H.-Q. Chu and L.-H. Jiang, "Correlation analysis between concrete parameters and electrodeposition effect based on grey theory," Journal of Wuhan University of Technology, vol. 31, no. 7, pp. 22-26, 2009.

[11] N. Otsuki, M. Hisada, J. S. Ryu, and E. J. Banshoya, "Rehabilitation of concrete cracks by electrodeposition," Concrete International, vol. 21, no. 3, pp. 58-62, 1999.

[12] J.-S. Ryu and N. Otsuki, "Crack closure of reinforced concrete by electrodeposition technique," Cement and Concrete Research, vol. 32, no. 1, pp. 159-164, 2002.

[13] Z. Jiang, F. Xing, Z. Sun, and P. Wang, "Healing effectiveness of cracks rehabilitation in reinforced concrete using electrodeposition method," Journal Wuhan University of Technology, Materials Science Edition, vol. 23, no. 6, pp. 917-922, 2008.

[14] Q. Chen, The stochastic micromechanical models of the multiphase materials and their applications for the concrete repaired by electrochemical deposition method [Ph.D. thesis], Tongji University, Shanghai, China, 2014.

[15] H. Zhu, Q. Chen, Z. Yan, J. W. Ju, and S. Zhou, "Micromechanical models for saturated concrete repaired by the electrochemical deposition method," Materials and Structures/Materiaux et Constructions, vol. 47, no. 6, pp. 1067-1082, 2014.

[16] Q. Chen, H. Zhu, Z. Yan, T. Deng, and S. Zhou, "Micro-scale description of saturated concrete repaired by electrochemical deposition method based on Mori-Tanaka method," Journal of Building Structures, vol. 36, no. 1, pp. 98-103, 2015.

[17] Q. Chen, H. H. Zhu, Z. G. Yan, J. W. Ju, T. Deng, and S. Zhou, "Micro-scale description of the saturated concrete repaired by electrochemical deposition method based on self-consistent method," Chinese Journal of Theoretical and Applied Mechanics, vol. 47, no. 2, pp. 367-371, 2015.
[18] Q. Chen, Z. W. Jiang, Z. H. Yang et al., "Differential-scheme based micromechanical framework for saturated concrete repaired by the electrochemical deposition method," Materials and Structures, vol. 49, no. 12, pp. 5183-5193, 2016.

[19] J. W. Ju and T. M. Chen, "Micromechanics and effective moduli of elastic composites containing randomly dispersed ellipsoidal inhomogeneities," Acta Mechanica, vol. 103, no. 1-4, pp. 103-121, 1994.

[20] T. Mura, Micromechanics of Defects in Solids, Martinus Nijhoff, The Hague, Netherlands, 1987.

[21] J. M. Qu and M. Cherkaoui, Fundamentals of Micromechanics of Solids, John Wiley \& Sons, Hoboken, NJ, USA, 2006.

[22] Q. Chen, H. H. Zhu, J. W. Ju et al., "A stochastic micromechanical model for multiphase composites containing spherical inhomogeneities," Acta Mechanica, vol. 226, no. 6, pp. 18611880, 2015.

[23] J. Qu, "Eshelby tensor for an elastic inclusion with slightly weakened interface," Journal of Applied Mechanics, vol. 60, no. 4, pp. 1048-1050, 1993.

[24] J. Qu, "The effect of slightly weakened interfaces on the overall elastic properties of composite materials," Mechanics of Materials, vol. 14, no. 4, pp. 269-281, 1993.

[25] K. Yanase and J. W. Ju, "Effective elastic moduli of spherical particle reinforced composites containing imperfect interfaces," International Journal of Damage Mechanics, vol. 21, no. 1, pp. 97127, 2012.

[26] S. H. Nie and C. Basaran, "A micromechanical model for effective elastic properties of particulate composites with imperfect interfacial bonds," International Journal of Solids and Structures, vol. 42, no. 14, pp. 4179-4191, 2005.

[27] Q. Chen, M. M. Nezhad, Q. Fisher, and H. H. Zhu, "Multiscale approach for modeling the transversely isotropic elastic properties of shale considering multi-inclusions and interfacial transition zone," International Journal of Rock Mechanics and Mining Sciences, vol. 84, pp. 95-104, 2016.

[28] Z. G. Yan, Q. Chen, H. H. Zhu, J. W. Ju, S. Zhou, and Z. W. Jiang, "A multi-phase micromechanical model for unsaturated concrete repaired using the electrochemical deposition method," International Journal of Solids and Structures, vol. 50, no. 24, pp. 3875-3885, 2013.

[29] G. Q. Li, Y. Zhao, and S.-S. Pang, "Four-phase sphere modeling of effective bulk modulus of concrete," Cement and Concrete Research, vol. 29, no. 6, pp. 839-845, 1999.

[30] N. B. Nguyen, A. Giraud, and D. Grgic, "A composite sphere assemblage model for porous oolitic rocks," International Journal of Rock Mechanics and Mining Sciences, vol. 48, no. 6, pp. 909-921, 2011.

[31] J. W. Ju and T. M. Chen, "Effective elastic moduli of twophase composites containing randomly dispersed spherical inhomogeneities," Acta Mechanica, vol. 103, no. 1-4, pp. 123-144, 1994.

[32] J. W. Ju and X. D. Zhang, "Micromechanics and effective transverse elastic moduli of composites with randomly located aligned circular fibers," International Journal of Solids and Structures, vol. 35, no. 9-10, pp. 941-960, 1998.

[33] J. W. Ju and L. Z. Sun, "A novel formulation for the exteriorpoint Eshelby's tensor of an ellipsoidal inclusion," Journal of Applied Mechanics, vol. 66, no. 2, pp. 570-574, 1999.

[34] J. W. Ju and L. Z. Sun, "Effective elastoplastic behavior of metal matrix composites containing randomly located aligned 
spheroidal inhomogeneities. Part I: micromechanics-based formulation," International Journal of Solids and Structures, vol. 38, no. 2, pp. 183-201, 2001.

[35] J. W. Ju and K. Yanase, "Micromechanics and effective elastic moduli of particle-reinforced composites with near-field particle interactions," Acta Mechanica, vol. 215, no. 1-4, pp. 135-153, 2010.

[36] J. W. Ju and K. Yanase, "Micromechanical effective elastic moduli of continuous fiber-reinforced composites with nearfield fiber interactions," Acta Mechanica, vol. 216, no. 1-4, pp. 87-103, 2011.

[37] L. Z. Sun and J. W. Ju, "Effective elastoplastic behavior of metal matrix composites containing randomly located aligned spheroidal inhomogeneities. Part II: applications," International Journal of Solids and Structures, vol. 38, no. 2, pp. 203-225, 2001.

[38] L. Z. Sun and J. W. Ju, "Elastoplastic modeling of metal matrix composites containing randomly located and oriented spheroidal particles," Journal of Applied Mechanics, vol. 71, no. 6, pp. 774-785, 2004.

[39] H. H. Zhu, Q. Chen, J. W. Ju et al., "Maximum entropy-based stochastic micromechanical model for a two-phase composite considering the inter-particle interaction effect," Acta Mechanica, vol. 226, no. 9, pp. 3069-3084, 2015.

[40] Q. Chen, H. H. Zhu, Z. G. Yan, J. W. Ju, Z. W. Jiang, and Y. Q. Wang, "A multiphase micromechanical model for hybrid fiber reinforced concrete considering the aggregate and ITZ effects," Construction and Building Materials, vol. 114, pp. 839-850, 2016.

[41] M. Mousavi Nezhad, H. H. Zhu, J. W. Ju, and Q. Chen, "A simplified multiscale damage model for the transversely isotropic shale rocks under tensile loading," International Journal of Damage Mechanics, vol. 25, no. 5, pp. 705-726, 2016.

[42] R. M. Christensen and K. H. Lo, "Solutions for effective shear properties in three phase sphere and cylinder models," Journal of the Mechanics and Physics of Solids, vol. 27, no. 4, pp. 315-330, 1979.

[43] H. L. Wang and Q. B. Li, "Prediction of elastic modulus and Poisson's ratio for unsaturated concrete," International Journal of Solids and Structures, vol. 44, no. 5, pp. 1370-1379, 2007.

[44] Z.-W. Jiang, W.-T. Li, Z.-L. Deng, and Z.-G. Yan, "Experimental investigation of the factors affecting accuracy and resolution of the pore structure of cement-based materials by thermoporometry," Journal of Zhejiang University: Science A, vol. 14, no. 10, pp. 720-730, 2013.

[45] Z. W. Jiang, Z. P. Sun, and P. M. Wang, "Internal relative humidity distribution in high-performance cement paste due to moisture diffusion and self-desiccation," Cement and Concrete Research, vol. 36, no. 2, pp. 320-325, 2006.

[46] Z. W. Jiang, Z. P. Sun, and P. M. Wang, "Autogenous relative humidity change and autogenous shrinkage of highperformance cement pastes," Cement and Concrete Research, vol. 35, no. 8, pp. 1539-1545, 2005.

[47] J. G. Berryman, "Long-wave propagation in composite elastic media II. Ellipsoidal inclusion," Journal of the Acoustical Society of America, vol. 68, no. 6, pp. 1820-1831, 1980.

[48] I. O. Yaman, N. Hearn, and H. M. Aktan, "Active and non-active porosity in concrete-part I: experimental evidence," Materials and Structures/Materiaux et Constructions, vol. 35, article 102, 2002.

[49] L. Cohen and O. Ishai, "The elastic properties of three-phase composites," Journal of Composite Materials, vol. 1, no. 4, pp. 390-403, 1967. 


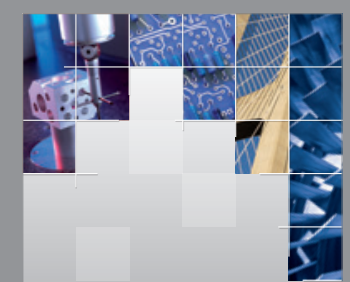

\section{Enfincering}
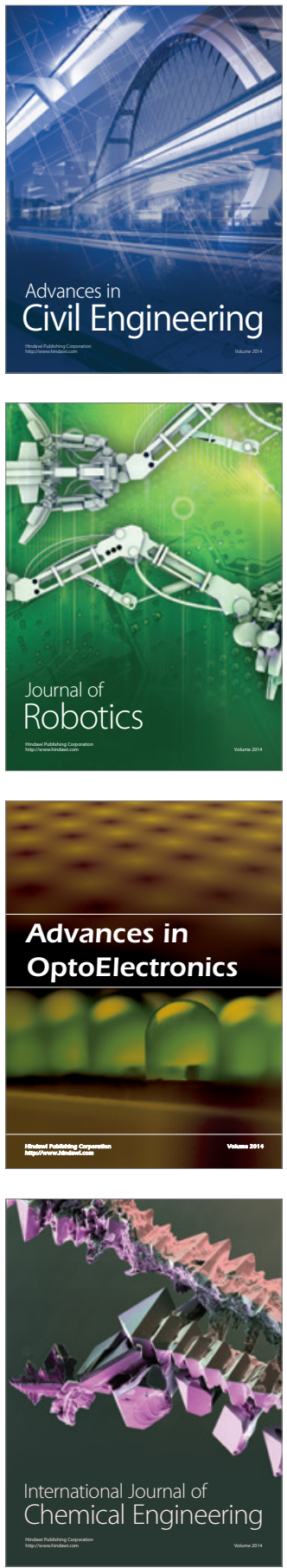

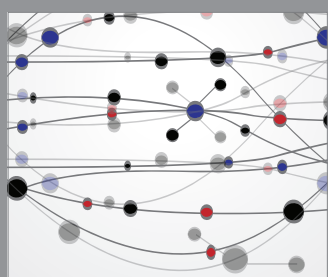

The Scientific World Journal

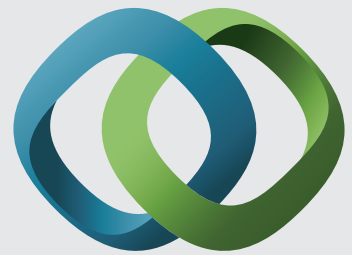

\section{Hindawi}

Submit your manuscripts at

http://www.hindawi.com
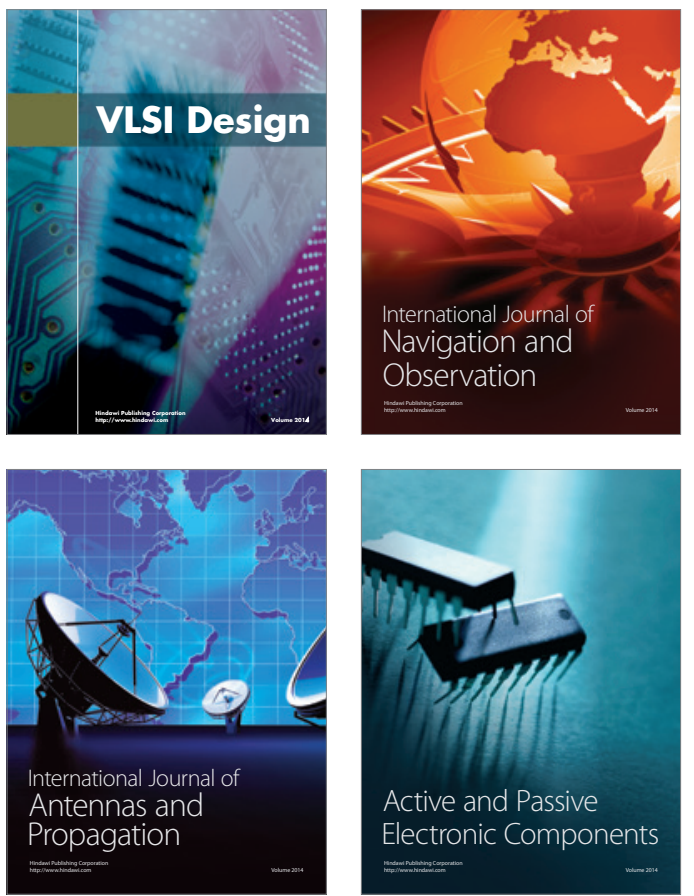
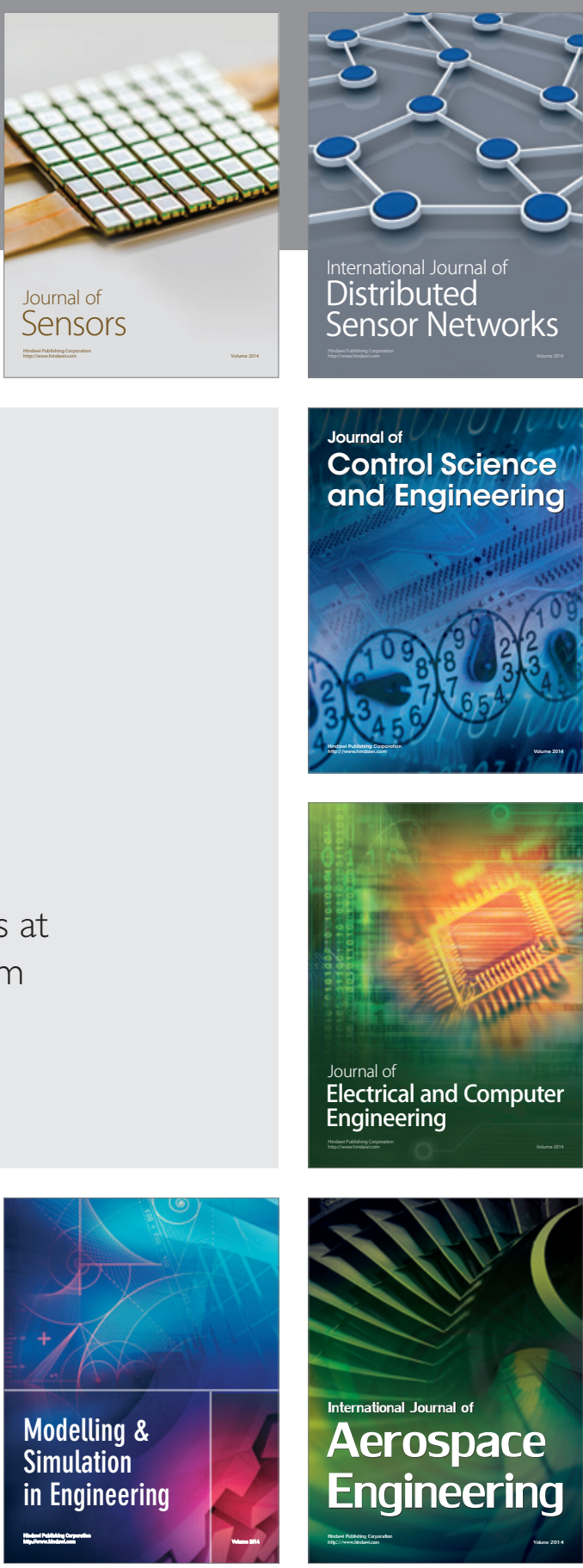

International Journal of

Distributed

Sensor Networks

Journal of

Control Science

and Engineering
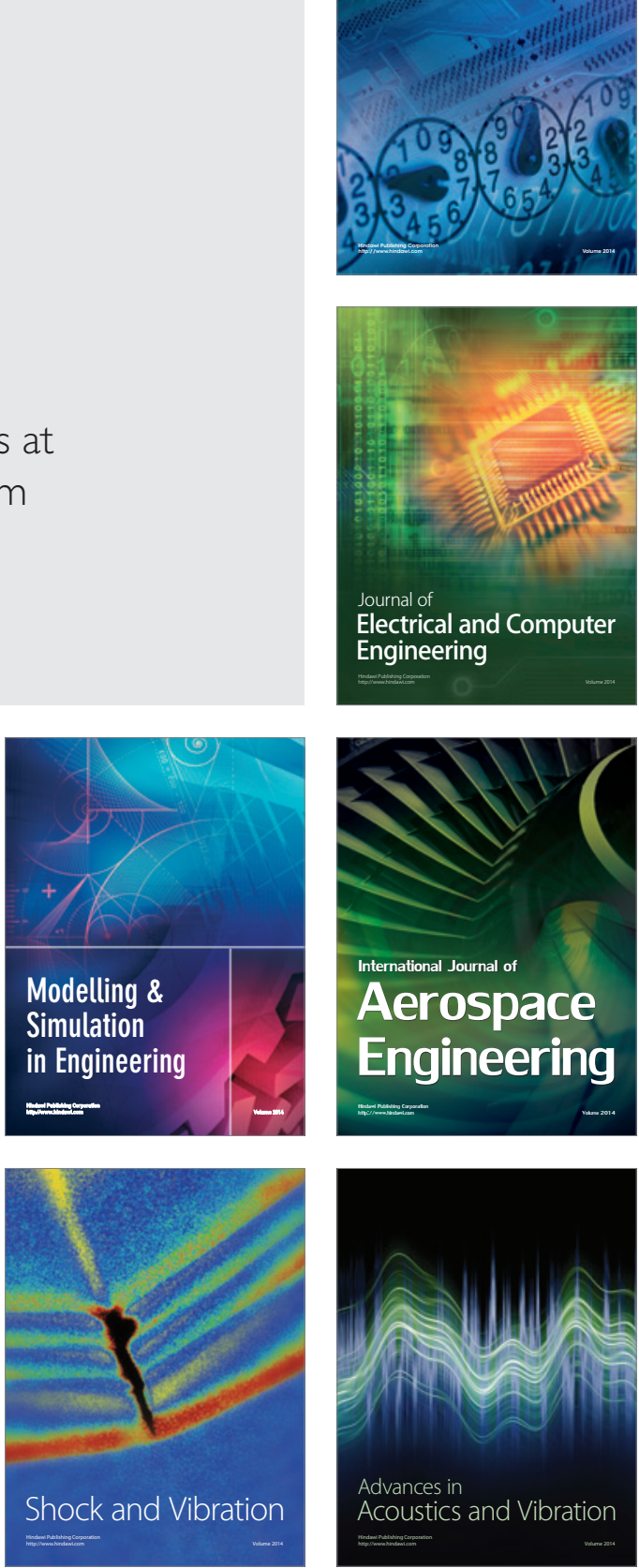